\title{
Development of circuit model of AC/coaxial adapter using for calibration of AMN
}

\author{
Ryosuke Tani ${ }^{1,2 a)}$, Ifong $\mathbf{W u}^{2}$, Kaoru Gotoh ${ }^{2}$, Yasushi Matsumoto ${ }^{2}$, \\ Shinobu Ishigami ${ }^{2,3 b)}$, Ryosuke Suga ${ }^{1 \mathrm{c})}$, and Osamu Hashimoto ${ }^{1}$ \\ ${ }^{1}$ College of Science and Engineering, Aoyama Gakuin University, \\ 5-10-1 Fuchinobe, Chuo-ku, Sagamihara-shi, Kanagawa 252-5258, Japan \\ ${ }^{2}$ National Institute of Information and Communications Technology, \\ 4-2-1 Nukui-kitamachi, Koganei-shi, Tokyo 184-8795, Japan \\ ${ }^{3}$ Tohoku Gakuin University, 1-13-1 Chuo, Tagajo-shi, Miyagi 985-8537, Japan \\ a)r-tani@ee.aoyama.ac.jp \\ b)shinobu@mail.tohoku-gakuin.ac.jp \\ c) rsuga@ee.aoyama.ac.jp
}

Abstract: Transmission characteristics of an AC/coaxial adapter that is used for calibration of an AMN had been evaluated up to $30 \mathrm{MHz}$, which is the upper limit of frequency in the use of conventional AMNs. However, the new type AMNs for using over $30 \mathrm{MHz}$ has been developed, thus the transmission characteristics of the adapter beyond $30 \mathrm{MHz}$ should be evaluated. In this paper, a circuit model of the AC/coaxial adapter is developed in order to obtain easily its transmission characteristics up to $1 \mathrm{GHz}$. The calculated characteristics using the developed circuit model agreed well with that of simulated by using a numerical electromagnetic analysis.

Keywords: AC/coaxial adapter, circuit model, artificial mains network Classification: Electromagnetic compatibility (EMC)

\section{References}

[1] Y. Matsumoto, I. Wu, K. Gotoh, and S. Ishigami, "Measurement and modeling of electromagnetic noise from LED light bulbs," IEEE Electromagn. Compat., vol. 2, no. 4, pp. 58-66, 2013. DOI:10.1109/MEMC.2013.6714699

[2] CISPR Publication 16-1-2 Ed. 2.0: Specification for radio disturbance and immunity measuring apparatus and methods-Part 1-2: Radio disturbance and immunity measuring apparatus-coupling devices for conducted disturbance measurements, 2014.

[3] T. Shinozuka, K. Fujii, A. Sugiura, and O. Wada, "Influence of an AC-coaxial adapter on measurements of the AMN impedance," IEICE Commun. Express, vol. 4, no. 3, pp. 99-104, Mar. 2015. DOI:10.1587/comex.4.99

[4] T. Shinozuka, K. Fujii, A. Sugiura, and O. Wada, "Calibration methods for AC-coaxial adapter used in AMN impedance measurements," IEEE Trans. Electromagn. Compat., vol. 58, no. 5, pp. 1388-1397, Oct. 2016. DOI:10.1109/ TEMC.2016.2582839 
[6] R. Tani, I. Wu, S. Ishigami, Y. Matsumoto, R. Suga, and O. Hashimoto, "Characteristic evaluation of conducted disturbance measuring apparatus using two parallel TEM cells," Proc. 2016 Int. Symp. Electromag, Compat., pp. 440-444, Sep. 2016. DOI:10.1109/EMCEurope.2016.7739231

[7] Japanese Industrial Standards JIS-C-8303, 2007 (in Japanese).

[8] C. R. Paul, Analysis of Multiconductor Transmission Lines, 2nd Ed., Chap. 7, pp. 314-316, John Wiley \& Sons, Inc., Hoboken, New Jersey, 2008.

\section{Introduction}

In recent years, energy-efficient equipment such as a power conditioner and LED bulbs have been rapidly spread by higher awareness for energy conservation. The energy is conserved by using a high-efficient power supply switching circuit in the equipment. However, the switching circuit often cause an electromagnetic disturbance over a wide frequency range from $\mathrm{kHz}$ to $\mathrm{GHz}$ [1]. Since this disturbance may interfere in the other electronics devices and broadcastings, the measurement of the disturbance is necessary.

An artificial mains network (AMN) [2] is used for measuring the conducted disturbance voltage below $30 \mathrm{MHz}$. Fig. 1(a) shows an example of conducted disturbance measurement using the AMN. As shown in the figure, an equipment under test (EUT) and a measuring receiver are connected to the AMN. The AMN supplies AC power to the EUT and transmits the disturbance occurring in the EUT to the measuring receiver. To obtain measurements result of the disturbance voltage, fundamental characteristics of the AMN such as the AMN impedance and the voltage division factor [2] need to be evaluated. Fig. 1(b) shows an example schema of AMN's characteristics measurement. As shown in the figure, the $\mathrm{AC} /$ coaxial adapter [3] is used for connecting the $\mathrm{AC}$ outlet of the $\mathrm{AMN}$ to a vector network analyzer. Thus, the measured characteristics of the AMN include the characteristics of the adapter. The characteristics of the adapter should be evaluated for obtaining the characteristics of the AMN without the adapter.

The characteristics of the adapter had been evaluated up to $30 \mathrm{MHz}$ which is the upper limit frequency of the conducted disturbance measurement using the conventional $\operatorname{AMN}[3,4,5]$. However, since the conducted disturbance measurement of over $30 \mathrm{MHz}$ is required by the above background, new type AMN for using up to $1 \mathrm{GHz}$ has been developed [6]. Therefore, the characteristics should be also evaluated beyond $30 \mathrm{MHz}$.

In this paper, we develop a new circuit model of the adapter for obtaining the transmission characteristics of the adapter beyond $30 \mathrm{MHz}$. Although the characteristics of the adapter can be also evaluated by using a 3D electromagnetic simulation, it is difficult to clarify the phenomenon from the result in the simulator. On the other hand, the circuit model can reduce a calculation cost for obtaining the characteristics and clarify the phenomenon. The characteristics obtained by the developed circuit model are compared with those of an electromagnetic simulation to clarify the validity of the developed circuit model. 


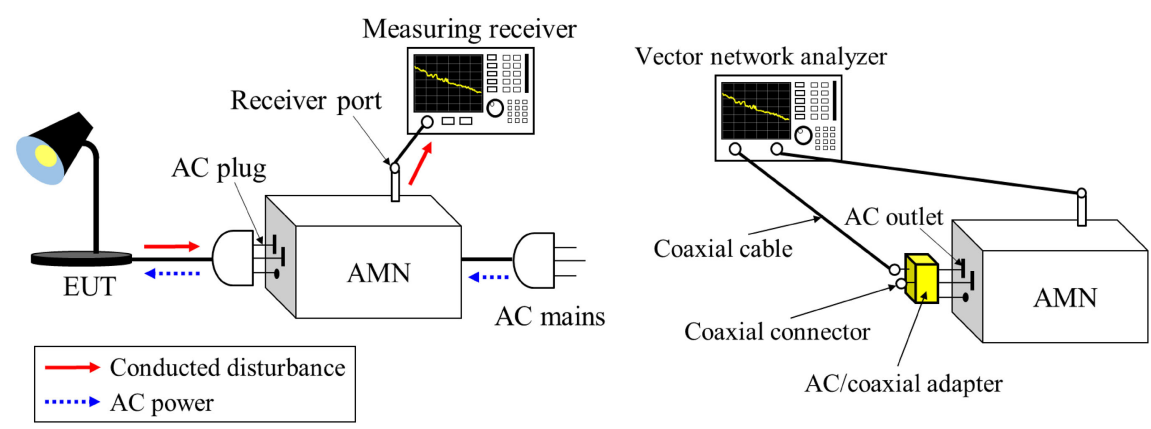

(a) Conducted disturbance measurement

(b) Characteristics measurement of AMN

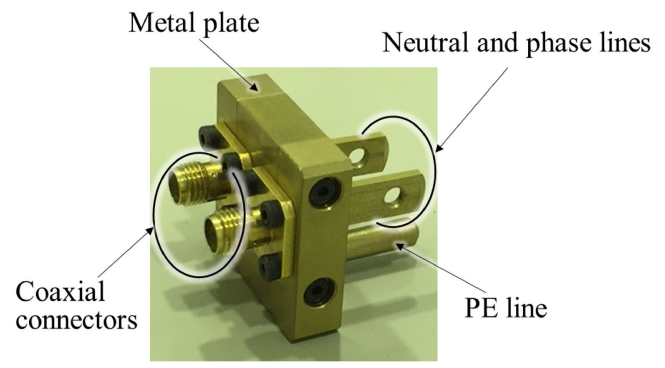

(c) An AC/coaxial adapter

Fig. 1. General outline of $\mathrm{AMN}$ and $\mathrm{AC} /$ coaxial adapter.

\section{Developed circuit model of AC/coaxial adapter}

\subsection{Structure}

Fig. 1(c) shows an AC/coaxial adapter. This adapter consists of two coaxial connectors, an AC plug, and a metal plate. The neutral and the phase lines connect directly each inner conductor of the connector. The protective earth (PE) line connects each outer conductors of the connector through the metal plate.

Fig. 2 shows the developed circuit model of the adapter. The AC plug can be treated as a transmission line because it consists of the conductors having uniform cross-section. There are three conductor lines $\# 1, \# 2$ and $\# 3$ above the infinite ground plane (GND) as shown in Fig. 2(a). The lines \#1 and \#2 represent the neutral and the phase lines, and the line \#3 represents the PE line. Input ports of the lines \#1, \#2 and \#3 are defined as port 1, 2, and 3, respectively. Output ports of the lines $\# 1, \# 2$ and $\# 3$ are defined as port 4,5 , and 6 , respectively. The dimensions of the cross-section shown in Fig. 2(b) meet the JIS standard [7], and " $h$ " is the height between the line \#3 and GND. The two adapters which are connected as a back-toback state will be measured because the ports of the adapter should be coaxial shape for connecting the vector network analyzer. Hence, a line length of the model is $34 \mathrm{~mm}$ which means the length of twice the AC plug. The equivalent circuit shown in Fig. 2(c) is lossless, and a telegrapher's equation of the circuit is given by Eq. (1).

$$
-\frac{d}{d x}\left[\begin{array}{c}
\boldsymbol{V}(x) \\
\boldsymbol{I}(x)
\end{array}\right]=j \omega\left[\begin{array}{ll}
\boldsymbol{O} & \boldsymbol{L} \\
\boldsymbol{C} & \boldsymbol{O}
\end{array}\right]\left[\begin{array}{c}
\boldsymbol{V}(x) \\
\boldsymbol{I}(x)
\end{array}\right]
$$

Here, $\boldsymbol{O}$ is a square zero matrix. $\boldsymbol{V}(x)$ and $\boldsymbol{I}(x)$ are vectors of line voltages and currents, respectively. $\boldsymbol{L}$ and $\boldsymbol{C}$ are inductance and capacitance matrices, respectively. 


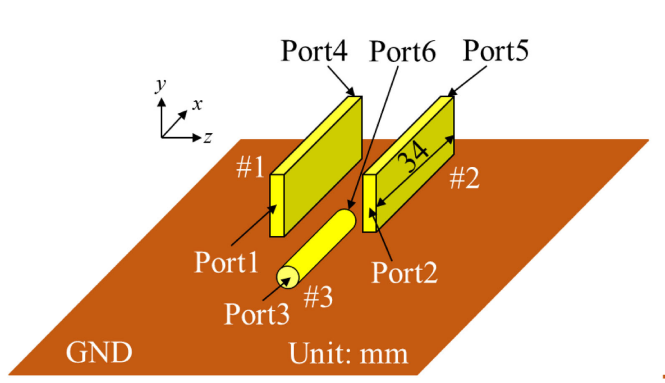

(a) Perspective view

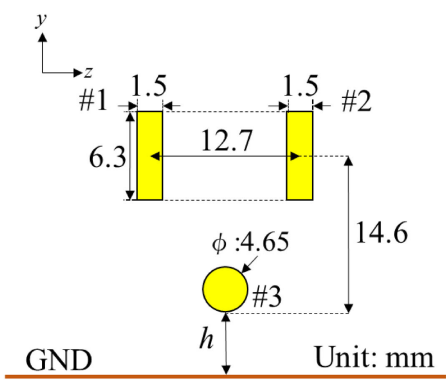

(b) Side view ( $y$-z plane)

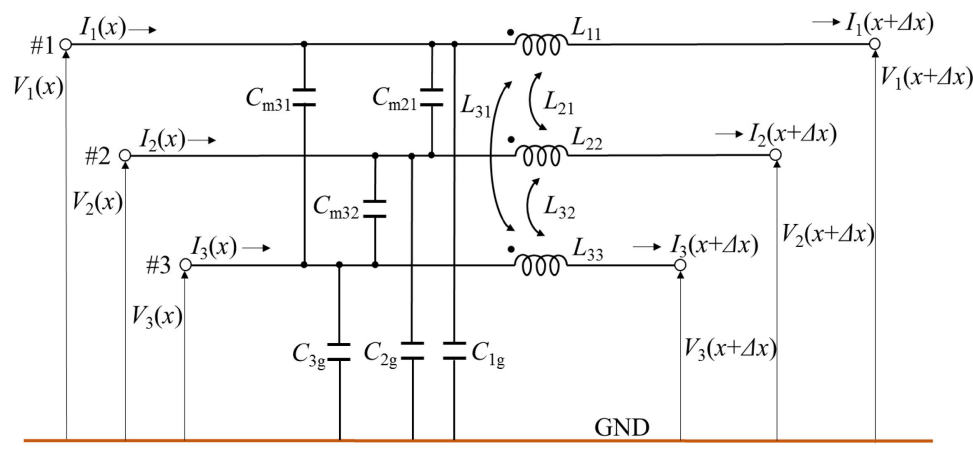

(c) Per-unit-length equivalent circuit

Fig. 2. The developed circuit model of the adapter.

\subsection{Evaluation method}

The F matrix of the AC plug $\boldsymbol{F}$ is derived from Eq. (1) using the state variable method [8]. Here, the values of $\boldsymbol{L}$ and $\boldsymbol{C}$ are analyzed by "ANSYS Q3D Extractor" which is the commercially-available code of parameter extraction. When each input port's voltages and currents are defined as $V_{1}, V_{2}, V_{3}, I_{1}, I_{2}$ and $I_{3}$, and each the output port's voltages and currents are defined as $V_{4}, V_{5}, V_{6}, I_{4}, I_{5}$ and $I_{6}$, the relation between the voltages and the currents are given by Eq. (2).

$$
\left[\begin{array}{l}
V_{1} \\
V_{2} \\
V_{3} \\
I_{1} \\
I_{2} \\
I_{3}
\end{array}\right]=\boldsymbol{F}\left[\begin{array}{l}
V_{4} \\
V_{5} \\
V_{6} \\
I_{4} \\
I_{5} \\
I_{6}
\end{array}\right]
$$

The adapter is excited by voltage difference between the neutral and PE lines or the phase and PE lines. The input voltages of the neutral and the phase lines are newly defined as $V_{1}^{\prime}$ and $V_{2}^{\prime}$, and the output voltages are also defined as $V_{3}^{\prime}$ and $V_{4}^{\prime}$. These defined voltages are given by Eq. (3).

$$
\begin{array}{ll}
V_{1}^{\prime}=V_{1}-V_{3}, & V_{2}^{\prime}=V_{2}-V_{3} \\
V_{3}^{\prime}=V_{4}-V_{6}, & V_{4}^{\prime}=V_{5}-V_{6}
\end{array}
$$

The currents which flow in the neutral and the phase lines return in the PE line. Therefore, the relation of the currents is shown Eq. (4)

$$
I_{1}+I_{2}=-I_{3}, \quad I_{4}+I_{5}=-I_{6}
$$


Then, a new F matrix $\boldsymbol{F}^{\prime}$ is derived by using Eqs. (3) and (4) that express the voltages and the currents conditions of the adapter. The relation between the voltages and currents using $\boldsymbol{F}^{\prime}$ are given by Eq. (5).

$$
\left[\begin{array}{c}
V_{1}^{\prime} \\
V_{2}^{\prime} \\
I_{1} \\
I_{2}
\end{array}\right]=\boldsymbol{F}^{\prime}\left[\begin{array}{c}
V_{3}^{\prime} \\
V^{\prime}{ }_{4} \\
I_{4} \\
I_{5}
\end{array}\right]
$$

The transmission characteristics of the adapter are evaluated by $\mathrm{S}$ matrix converted from $\boldsymbol{F}^{\prime}$.

\section{Validation of developed circuit model}

Fig. 3(a) shows an analysis model of the AC/coaxial adapters which are connected as a back-to-back state. All conductors of the analysis model are considered as perfect electric conductors. The dimensions of the coaxial connector are designed so that its characteristic impedance is $50 \mathrm{Ohm}$, and that of $\mathrm{AC}$ plugs are the same as the developed circuit model mentioned in Section 2. Also, the two inner conductors of the connectors are extended to avoid contact of the metal plate with the neutral/ phase lines as shown in Fig. 3(a). The 4-port S-parameters of the analysis model are numerically simulated by using the 3D electromagnetic simulator "HFSS", in a case of changing the height " $h$ " between the PE line and the GND.

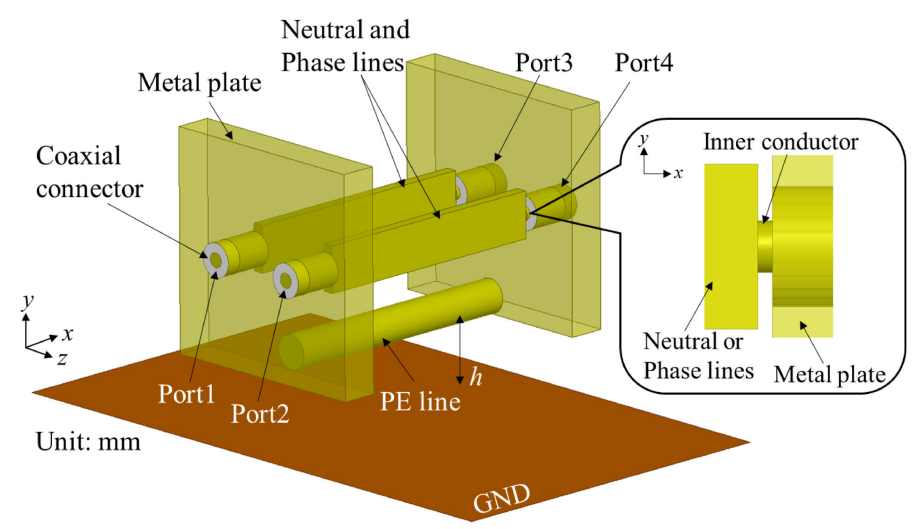

(a) An analysis model the adapters which are connected as a back-to-back state

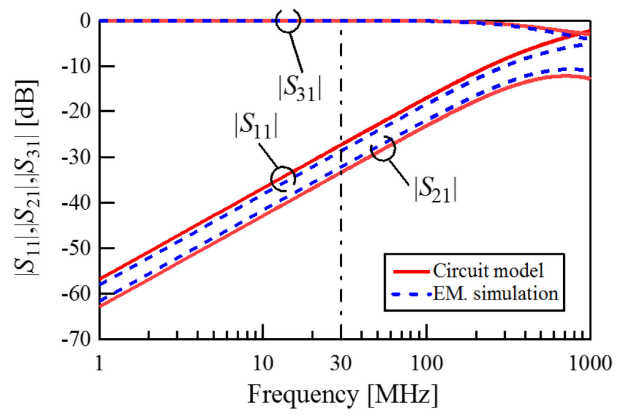

(b) S-parameters at $h=10 \mathrm{~mm}$

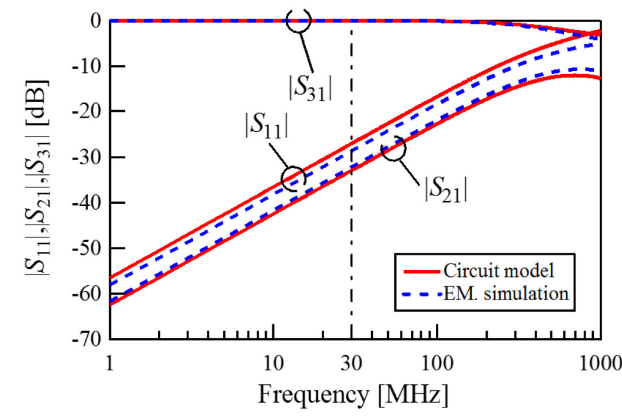

(c) S-parameters at $h=50 \mathrm{~mm}$

Fig. 3. The calculated S-parameters of the adapter using the developed circuit model. 
Fig. 3(b) and (c) show the calculated S-parameters of the adapter using the developed circuit model in cases of $h=10 \mathrm{~mm}$ and $50 \mathrm{~mm}$. As shown in the figures, the calculated characteristics using the model agree with the simulated one using the electromagnetic simulation regardless of $h$. This result shows the validity of the developed circuit model. The transmission characteristic $\left|S_{31}\right|$ of the adapter keeps $0 \mathrm{~dB}$ in less than $30 \mathrm{MHz}$. Thus, the adapter affects hardly the characteristics of the conventional AMN. However, the characteristic $\left|S_{31}\right|$ decreases in a case where the frequency exceeds $30 \mathrm{MHz}$ by increasing the reflection and the coupling between the neutral/phase lines. It is found that the adapter affect the characteristics of the new type AMN for using over $30 \mathrm{MHz}$.

\section{Conclusions}

In this paper, the circuit model of the adapter was developed for obtaining easily its transmission characteristics. The calculated S-parameters of the adapter using the developed circuit model were compared with the simulated one using the electromagnetic simulator. As a result, both S-parameters agreed well regardless of the height between the adapter and the GND. Therefore, the validity of the developed circuit model was indicated. Furthermore, it was found that the adapter affects the characteristics of the new type AMN for using over $30 \mathrm{MHz}$ because the transmission characteristics of the adapter decrease due to its reflection and coupling characteristics. In future works, a balance and an impedance of the adapter will be evaluated by using the developed circuit model, and an influence of the adapter for the AMN will be evaluated.

\section{Acknowledgments}

This work is partially supported by MEXT*-Supported Program for the Strategic Research Foundation at Private Universities, 2013-2017 (*Ministry of Education, Culture, Sports, Science and Technology). 